ESAIM: PROCEEDINGS AND SURVEYS, March 2016, Vol. 53, p. 22-37

M. Campos Pinto and F. Charles, Editors

\title{
MODELLING AND SIMULATING A MULTISPECIES PLASMA
}

\author{
Mehdi BADSI ${ }^{1}$ AND MaXime Herda ${ }^{2}$
}

\begin{abstract}
This paper is devoted to the modelling and numerical simulations of collisionless multispecies plasmas. In the framework of tokamak applications, we detail the dimensional analysis of the coupled kinetic system in order to extract the important parameters of the model. From there, we focus on two asymptotics. We first investigate the implementation of a solver adapted the quasineutral limit in order to deal with large scale simulations. In a second time, we study the massless electron approximation through numerical experiments and question the validity of the usual Maxwell-Boltzmann density approximation.
\end{abstract}

KEYWORDS. Vlasov-Poisson, multispecies plasma, tokamak plasma, numerical simulations.

Résumé. Cet article est consacré à la modélisation et la simulation numérique de plasmas multiespèces non-collisionnels. Dans le contexte des applications aux plasmas de tokamak, nous détaillons l'adimensionnement du système cinétique couplé afin d'en extraire les paramètres importants du modèle. De là, nous nous concentrons sur deux asymptotiques. Nous étudions d'abord la mise en œuvre d'un solveur adapté la limite quasineutre afin de pouvoir traiter des simulations à échelle macroscopique. Dans un second temps, nous étudions l'approximation des électrons sans masse à travers des expériences numériques et remettons en question la validité de l'approximation habituelle de densité de Maxwell-Boltzmann.

KEYWORDs. Vlasov-Poisson, multi-espèces, plasma de tokamak, simulations numériques.

\section{INTRODUCTION}

When a gas is brought to a high temperature, electrons leave their orbit around the nuclei. By becoming a mixture of charged particles, the matter enters a new state, called plasma. While the thermodynamical conditions on Earth prevent its existence, more than $99 \%$ of the visible universe is made of plasma. When the particles are closely confined, like in the stars because of the gravitational force, the nuclei of light atoms collide and produce energy. The reconstruction of this phenomenon of nuclear fusion in magnetic confinement devices such as tokamaks is one of the major energy challenges of the $21^{\text {st }}$ century. A fine control of the particles is required for their proper functioning can only be made possible by the detailed understanding of the dynamic of the plasma. The physical and mathematical study of associated models and the design of efficient numerical schemes is hence at the heart of this problem.

The aim of this work consists in investigating the numerical simulations of multispecies coupled kinetic models arising in plasma physics. The main complexity is to deal with the inner stiffness of the equations. Indeed, each species (electrons or ions) brings its own variety of spatio-temporal scales which makes it tricky to numerically

\footnotetext{
1 Laboratoire Jaques Louis-Lions (LJLL), Université Pierre et Marie Curie (Paris-VI), 4 place Jussieu, 75252 Paris Cedex 05, France. Email: badsi@ljll.math.upmc.fr

2 Institut Camille Jordan (ICJ), Université Claude Bernard Lyon 1, 43 boulevard du 11 novembre 1918 , 69622 Villeurbanne Cedex, France. Email: herda@math.univ-lyon1.fr 
describe the whole dynamic. Besides, the smallness of some physical parameters, such as the Debye length in the quasineutral regime, brings some additional issues to the conception of consistent schemes.

In this paper, we present the work done for the Multisplash project (Multispecies plasma simulations) during the CEMRACS 2014. The outline is as follows. In the first Section, we discuss the modelling. After presenting the equations in a general context, we perform a dimensional analysis in order to highlight the important parameters of the model. The goal of the rest of the paper is to study numerically two asymptotics associated with these parameters. Indeed, in the framework of a simplified one dimensional model, we will present in Section 2 the numerical methods implemented to perform the simulations. In particular we describe an asymptotic preserving numerical scheme dealing with the quasineutral approximation originally proposed by Belaouar, Crouseilles, Degond and Sonnendrücker [1] and adapt it here to our model. In the third section, we focus on the massless electron asymptotics. We make the mass ratio tends to zero and compare the density of electrons obtained for two species simulations with the usual Maxwell-Boltzmann density approximating the latter in most one species simulations.

\section{Modelling of A multispecies plasma}

\subsection{The physical modelling}

We consider a plasma constituted of ions and electrons. Because of their charge, the particles create their own electromagnetic field acting on one another. Hence the plasma can be modelled by a collection of particles in interaction. The description of the motion of each particle is possible thanks to the equations of Newton's dynamics. However, since the number of particles in a plasma is typically very large, a statistical or mesoscopic approach is more appropriate. The goal is not to follow each particle but to describe the evolution of the particle density in the phase space.

To avoid unnecessary technicalities, we suppose that there is only one type of ion in the plasma of mass $m_{i}$ and charge $q$, while $m_{e}$ denotes the mass of the electrons which have a negative charge of $-q$. The number of particle of type $\alpha$ at position $x \in \mathbb{R}^{3}$, with velocity $v \in \mathbb{R}^{3}$ between time $t$ and $t+d t$ is $f_{\alpha}(t, x, v) d x d v d t$. The index $\alpha$ stands for the species of the particles and can be either $i$ for ions or $e$ for electrons.

In the physical regime we are modelling, only the electrostatic effect are taken into account, which means that the self-induced magnetic field is neglected. This is a valid approximation as soon as the typical velocity of the particles is small compared to the velocity of light. However, we consider the effect of an external magnetic field. This configuration is typical of a tokamak plasma where the magnetic field is used to confine the particles inside the core of the device.

Each density $f_{\alpha}$ evolves according to a Vlasov-type equation and the coupling is done with the Poisson equation that links the electric field and the densities. On the time scale we will be dealing with, we suppose that the collisions between particles can be neglected. This is a common and justified approximation for tokamak plasmas (see [14]). The equations of the model, written in physical units, are the following,

$$
\left\{\begin{array}{l}
\partial_{t} f_{i}+v \cdot \nabla_{x} f_{i}+\frac{q}{m_{i}}\left(-\nabla_{x} \phi+v \wedge B_{\mathrm{ext}}\right) \cdot \nabla_{v} f_{i}=0, \\
\partial_{t} f_{e}+v \cdot \nabla_{x} f_{e}-\frac{q}{m_{e}}\left(-\nabla_{x} \phi+v \wedge B_{\mathrm{ext}}\right) \cdot \nabla_{v} f_{e}=0, \\
-\varepsilon_{0} \Delta_{x} \phi=q\left(n_{i}-n_{e}\right),
\end{array}\right.
$$

where $\varepsilon_{0}$ is the dielectric constant, $\phi$ the electric potential and $B_{\text {ext }}$ an external magnetic field. The coupling between the two kinetic equations is done through the Poisson equation which involves the macroscopic densities,

$$
n_{\alpha}=\int_{\mathbb{R}^{3}} f_{\alpha} d v, \quad \forall \alpha \in\{i, e\} .
$$


The dynamic of such coupled two-species model is very heterogeneous because of the smallness of the mass ratio $m_{e} / m_{i}$. The typical time scales of the ions and the electrons differ from several orders of magnitude. That explains the usual necessity for model reduction. Indeed, in the applications involving magnetic confinement fusion, the ions are the particles of interest and approximations are made on the electron density $f_{e}$ in order to simplify the model. A common hypothesis is to suppose that the macroscopic electronic density is given by the Maxwell-Boltzmann density,

$$
n_{\mathrm{MB}}(t, x)=C(t) e^{\frac{q \phi(t, x)}{k_{B} \theta}},
$$

where $C(t)$ is a normalization function. The derivation of the latter, with $B_{\text {ext }}=0$, is evoked in [5] and treated in [4] for a one species Vlasov-Poisson-Fokker-Planck model. In Section 3, we perform numerical experiments to compare two species simulations with this approximation in a stable and an unstable test case.

\subsection{Scaling}

We denote by $L$ the characteristic length of the system, $t_{0}$ the characteristic time and $V_{\alpha}$ the thermal velocity for the species $\alpha \in\{i, e\}$. For the physical quantity $G$, we denote by $\bar{G}$ the characteristic value of $G$ and $G^{\prime}$ the dimensionless quantity associated to $G$ such that $G=\bar{G} G^{\prime}$. Moreover we assume that the plasma is globally neutral, which means that

$$
\bar{n}_{i}=\bar{n}_{e}=: N
$$

and that the characteristic temperatures (or kinetic energy) of each species are equal. A plasma satisfying this last hypothesis is called a hot plasma [2] and satisfies with our notation

$$
m_{i} V_{i}^{2}=m_{e} V_{e}^{2}=k_{B} \theta
$$

The new unknowns of the system are then defined by the following relations,

$$
f_{\alpha}(t, x, v)=\frac{N}{V_{\alpha}^{3}} f_{\alpha}^{\prime}\left(\frac{t}{t_{0}}, \frac{x}{L}, \frac{v}{V_{\alpha}}\right), \quad n_{\alpha}(t, x)=N n_{\alpha}^{\prime}\left(\frac{t}{t_{0}}, \frac{x}{L}\right), \quad \phi(t, x)=\bar{\phi} \phi^{\prime}\left(\frac{t}{t_{0}}, \frac{x}{L}\right) .
$$

Introducing the characteristic quantities in the Vlasov-Poisson system (1.1) yields,

$$
\begin{aligned}
& \partial_{t^{\prime}} f_{i}^{\prime}+\frac{\bar{V}_{i} t_{0}}{L} v^{\prime} \cdot \nabla_{x^{\prime}} f_{i}^{\prime}+\frac{q t_{0}}{m_{i} V_{i}}\left(-\frac{\bar{\phi}}{L} \nabla_{x^{\prime}} \phi^{\prime}+V_{i} \bar{B} v^{\prime} \wedge B^{\prime}\right) \cdot \nabla_{v^{\prime}} f_{i}^{\prime}=0 \\
& \partial_{t^{\prime}} f_{e}^{\prime}+\frac{\overline{V_{e}} t_{0}}{L} v^{\prime} \cdot \nabla_{x^{\prime}} f_{e}^{\prime}-\frac{q t_{0}}{m_{i} V_{e}}\left(-\frac{\bar{\phi}}{L} \nabla_{x^{\prime}} \phi^{\prime}+V_{e} \bar{B} v^{\prime} \wedge B^{\prime}\right) \cdot \nabla_{v^{\prime}} f_{e}^{\prime}=0 \\
& -\frac{\varepsilon_{0} \bar{\phi}}{q N \bar{L}^{2}} \Delta_{x^{\prime}} \phi^{\prime}=n_{i}^{\prime}-n_{e}^{\prime} .
\end{aligned}
$$

The dimensional analysis of (1.1) introduces several important physical constant of the system, namely, for each species $\alpha \in\{i, e\}$,

$$
\lambda_{D}=\sqrt{\frac{\varepsilon_{0} k_{B} \theta}{q^{2} N}}, \quad t_{p}^{(\alpha)}=\frac{\lambda_{D}}{V_{\alpha}}, \quad t_{c}^{(\alpha)}=\frac{m_{\alpha}}{q \bar{B}_{\text {ext }}}, \quad r_{L}^{(\alpha)}=V_{\alpha} t_{c}^{(\alpha)},
$$

which are respectively the Debye length, the plasma time, the cyclotron time and the Larmor radius. More details on these constants can be found in the physics literature (see [2,14]). Let us just mention that the first two are the typical scales of the electrostatic effects while the last two are related to the magnetic phenomena. In the context of tokamak plasma simulations, we choose a scaling relative to the typical time scale of the ions. In particular, it means that,

$$
t_{0}=\frac{L}{V_{i}}
$$


From some of the physical constants and characteristic quantities arise the dimensionless parameters of the system,

$$
\delta=\frac{\lambda_{D}}{L}, \quad \eta=\frac{q \bar{\phi}}{k_{B} \theta}, \quad \mu=\frac{\bar{\phi}}{L V_{i} \bar{B}_{\mathrm{ext}}}, \quad \varepsilon=\sqrt{\frac{m_{e}}{m_{i}}} .
$$

For a tokamak plasma these parameters would all be small. Dealing with the asymptotic $\delta \rightarrow 0$ is called the quasineutral limit and has been studied for the Vlasov-Poisson system by Han-Kwan in [12]. The second parameter $\eta$ is called the coupling parameter, for it measures the importance of the electrostatic effects with respect to the thermal agitation. The third parameter $\mu$ compares the Coulomb (electric) and the Laplace (magnetic) forces. The limit $\mu \rightarrow 0$ corresponds to the case of a strong magnetic field and has been studied for single-species plasma by Frenod and Sonnendrucker in [9] and Golse and Saint-Raymond in [10,16]. This asymptotic is called the gyrokinetic approximation in the mathematics literature. The last parameter $\varepsilon$, quantifying the mass ratio and the limit $\varepsilon \rightarrow 0$ is called the massless electrons approximation. The dimensionless equations write,

$$
\left\{\begin{array}{l}
\partial_{t} f_{i}^{\prime}+v \cdot \nabla_{x} f_{i}^{\prime}-\eta \nabla_{x} \phi^{\prime} \cdot \nabla_{v} f_{i}^{\prime}+\frac{\eta}{\mu}\left(v \wedge B_{\mathrm{ext}}\right) \cdot \nabla_{v} f_{i}^{\prime}=0 \\
\partial_{t} f_{e}^{\prime}+\frac{1}{\varepsilon} v \cdot \nabla_{x} f_{e}^{\prime}+\frac{\eta}{\varepsilon} \nabla_{x} \phi^{\prime} \cdot \nabla_{v} f_{e}^{\prime}-\frac{\eta}{\mu \varepsilon^{2}}\left(v \wedge B_{\mathrm{ext}}\right) \cdot \nabla_{v} f_{e}^{\prime}=0 \\
-\eta \delta^{2} \Delta_{x} \phi^{\prime}=n_{i}^{\prime}-n_{e}^{\prime}
\end{array}\right.
$$

The link between the different time scales and the dimensionless parameters follows from the previous relations and writes,

$$
t_{p}^{i}=\frac{1}{\varepsilon^{2}} t_{p}^{e}=\delta t_{0}, \quad t_{c}^{i}=\frac{1}{\varepsilon} t_{c}^{e}=\frac{\mu}{\eta} t_{0}
$$

\subsection{The one dimensional case}

From now on, we assume that $\eta=1, B_{\text {ext }}=0$ and the system is formulated in dimension one. The choice of the coupling parameter is motivated by the fact that without magnetic field one can make the change of variable $\tilde{E}:=\eta E$ or equivalently set $\eta=1$. The rescaled two species Vlasov-Poisson system writes, dropping the primes,

$$
\left\{\begin{array}{l}
\partial_{t} f_{i}+v \partial_{x} f_{i}+E \partial_{v} f_{i}=0 \\
\partial_{t} f_{e}+\frac{1}{\varepsilon} v \partial_{x} f_{e}-\frac{1}{\varepsilon} E \partial_{v} f_{e}=0 \\
\delta^{2} \partial_{x} E=n_{i}-n_{e}
\end{array}\right.
$$

where,

$$
E=-\partial_{x} \phi
$$

is the electric field associated with the potential $\phi$. The velocity $v$ varies on the whole real line $\mathbb{R}$ while the space domain is periodic of length $L$, i.e. $x \in \mathbb{T}_{L}=\mathbb{R} / L \mathbb{Z}$. Let us recall some important features of this model. Under compatible assumptions on the initial data the following properties hold for classical solutions of (1.4) (see [6] for more details), for $\alpha \in\{i, e\}$,

\section{Positivity:}

$$
f_{\alpha} \geq 0
$$

\section{$L^{p}$ norm conservation:}

$$
\frac{d}{d t} \iint_{\mathbb{R} \times \mathbb{T}_{\mathbb{L}}} f_{\alpha}^{p} d v d x=0, \quad \forall p \in[1,+\infty] .
$$




\section{Energy conservation:}

$$
\frac{d}{d t} \mathcal{E}=\frac{d}{d t}\left[\frac{1}{2} \iint_{\mathbb{R} \times \mathbb{T}_{\mathbb{L}}}|v|^{2}\left(f_{i}+f_{e}\right) d v d x+\frac{1}{2} \int_{\mathbb{T}_{\mathbb{L}}}|E|^{2} d x\right]=0 .
$$

\section{Entropy conservation:}

$$
\frac{d}{d t} \mathcal{S}_{\alpha}=\frac{d}{d t} \iint_{\mathbb{R} \times \mathbb{T}_{\mathbb{L}}} f_{\alpha} \ln f_{\alpha} d v d x=0
$$

Moreover, for initial data $f_{i}^{\text {in }}, f_{e}^{\text {in }}$, a smooth solution writes, for $\alpha \in\{i, e\}$,

$$
f_{\alpha}(t, x, v)=f_{\alpha}^{\text {in }}\left(X_{\alpha}(0 ; t, x), V_{\alpha}(0 ; t, v)\right),
$$

where $X_{\alpha}$ and $V_{\alpha}$ solve the characteristics associated with the system (1.4),

$$
\left\{\begin{array} { l } 
{ \frac { d X _ { i } } { d s } ( s ; t , x ) = V _ { i } ( s ; t , v ) , } \\
{ \frac { d V _ { i } } { d s } ( s ; t , v ) = E ( t , X _ { i } ( s ; t , x ) ) , }
\end{array} \quad \left\{\begin{array}{l}
\frac{d X_{e}}{d s}(s ; t, x)=\frac{1}{\varepsilon} V_{e}(s ; t, v), \\
\frac{d V_{e}}{d s}(s ; t, v)=-\frac{1}{\varepsilon} E\left(t, X_{e}(s ; t, x)\right),
\end{array}\right.\right.
$$

and, for $\alpha \in\{i, e\}$,

$$
X_{\alpha}(t ; t, x)=x \quad V_{\alpha}(t ; t, v)=v .
$$

\section{Numerical Methods}

In this section, we present the numerical methods implemented to solve (1.4). After a brief state of the art, we describe the backward semi-Lagrangian method used for the resolution of the Vlasov equations and adapt an asymptotic preserving scheme for the Poisson equation in the quasineutral regime $\delta \ll 1$. The latter was introduced in [1] and we shall detail its implementation in our model. However, we left its numerical validation with respect to both the massless electron limit and quasineutral regime for future work.

\subsection{State of the art}

The accurate simulation of the Vlasov equation is a delicate problem. Indeed the system has some inherent properties that must be preserved in order to describe the physics underneath the equations. Namely, the invariants such as the mass, the energy or the $L^{p}$ norms must be preserved by the scheme. Consequently, either the latter is designed to naturally conserve these macroscopic quantities, or they are used as control variates for the simulation. In this context of multispecies simulations, the stiffness towards the parameters $\varepsilon$ and $\delta$ makes it even harder to fulfil these objectives.

There are two major categories of numerical methods for solving the Vlasov equation. The first and oldest, is the category of particle methods such as the Particle-In-Cell method. Its principle is based on the statistical description of the origin of the Vlasov equation. It is a Monte-Carlo type method. One approaches the initial densities with a large number of particles which trajectories are followed in time with the characteristic ODEs. The literature on the subject is vast and includes a more physical description of the method in Birdsall and Langdon [3] and mathematical proofs of convergence in Neunzert and Wick [15]. PIC methods still serve as benchmark in terms of full $6 \mathrm{D}$ simulations of Vlasov equations because by construction, the computational cost depends weakly on the dimension. Only the complexity of fields reconstruction increases drastically with the dimension of the physical space.

The second category consists of methods known as Eulerian, including the semi-Lagrangian, finite volume, Galerkin or finite difference methods. Eulerian schemes aim at solving the equations on a fixed discretization of the phase space. A comparison of some of these methods for the one species Vlasov-Poisson system can be 
found in the survey of Filbet and Sonnendrücker [8]. We adapt a backward semi-Lagrangian code to our two species model.

\subsection{The Backward Semi-Lagrangian method}

The semi-Lagrangian method is based on the fact, coming from equation (1.5), that the densities $f_{i}$ and $f_{e}$ are constant along their characteristics. Assuming that the densities are known at time $t^{n}=n \Delta t$ then we get their value at time $t^{n+1}$ by the following relations, for $\alpha \in\{i, e\}$,

$$
f_{\alpha}\left(t^{n+1}, x, v\right)=f_{\alpha}\left(t^{n}, X_{\alpha}\left(t^{n} ; t^{n+1}, x\right), V_{\alpha}\left(t^{n} ; t^{n+1}, v\right)\right) .
$$

One goes one step back in time and evaluates the densities at the feet of the characteristics. To solve the characteristics, we use here a classical Strang splitting technique that allows us, in this case, to approximate the characteristics with an explicit formula of order 2 in time.

Remark 2.1. For our one dimensional unmagnetized model, the explicit formula for the characteristics allows us to handle small values of $\varepsilon$ without having to numerically solve a stiff ODE. However, to ensure a uniform approximation for the study of the $\varepsilon \rightarrow 0$ limit, one should take a time step such that $\Delta t=o(\sqrt{\varepsilon})$.

A sketch of the principle can be found on Figure 1 for a cartesian mesh in the phase space $\left(x_{i}, v_{j}\right)_{i \in I, j \in J}$. In

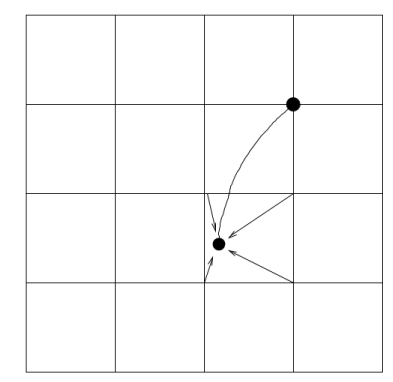

FIGURE 1. Reconstruction phase for the backward semi-Lagrangian method

general, the characteristics feet don't coincide with the mesh points. Therefore, a reconstruction of the densities by interpolation is required. A wide literature exists on this important phase. Let us just mention that in our case we used a high order Hermite weighted essentially non oscillatory (HWENO) method designed by Filbet and Yang [17]. This method gives an excellent accuracy because of the order of interpolation while avoiding the drawback of numerical oscillations.

The introduction of the mesh brings the question of the boundary conditions. In space, system (1.4) is set on the torus of length $L$. Consequently, we consider periodic boundary conditions for the $\left(x_{i}\right)_{i \in I}$. The velocity variable is defined on the whole real line for the continuous problem. However, we restrain it to a segment $[-V, V]$ with $V$ large enough to keep the main part of the mass. This is possible since the typical densities quickly decrease in $|v|$ at infinity. In other words most of the particles don't go too fast. Therefore we associate homogeneous Dirichlet conditions for the boundary velocities.

\subsection{An asymptotic preserving scheme for the Poisson equation in the quasineutral limit}

In dimension 1 , it is trivial to solve numerically the Poisson equation,

$$
\delta^{2} \partial_{x} E=n_{i}-n_{e},
$$

from the knowledge of the macroscopic densities, by a direct numerical integration. However, with this method the error blows up when the quasineutrality parameter $\delta$ tends to 0 . This comes directly from the continuous 
problem. Indeed the formal limit of the Poisson equation is reduced to the quasineutrality constraint $n_{i}=n_{e}$. Namely, we lose the ability to compute the potential or the electric field. In order to propose an asymptotic preserving scheme to manage small values of $\delta$, the first step is to derive a reformulated Poisson equation adapted to the quasineutral limit.

By multiplying the Vlasov equations of (1.4) by 1 and $v$ and integrating in the $v$ variable, one gets the following moment equations,

$$
\begin{aligned}
& \partial_{t} n_{i}+\partial_{x} j_{i}=0, \\
& \partial_{t} n_{e}+\frac{1}{\varepsilon} \partial_{x} j_{e}=0, \\
& \partial_{t} j_{i}+\partial_{x} S_{i}=-n_{i} \partial_{x} \phi, \\
& \partial_{t} j_{e}+\frac{1}{\varepsilon} \partial_{x} S_{e}=\frac{n_{e}}{\varepsilon} \partial_{x} \phi .
\end{aligned}
$$

where the first order moments write, for $\alpha \in\{i, e\}$,

$$
j_{\alpha}=\int v f_{\alpha} d v \quad S_{\alpha}=\int v^{2} f_{\alpha} d v
$$

Subtracting (2.1) with (2.2) and (2.3) with (2.4) divided by $\varepsilon$, one gets respectively,

$$
\begin{aligned}
& \partial_{t}\left(n_{i}-n_{e}\right)+\partial_{x}\left(j_{i}-\frac{j_{e}}{\varepsilon}\right)=0, \\
& \partial_{t}\left(j_{i}-\frac{j_{e}}{\varepsilon}\right)+\partial_{x}\left(S_{i}-\frac{S_{e}}{\varepsilon^{2}}\right)=-\left(n_{i}+\frac{n_{e}}{\varepsilon^{2}}\right) \partial_{x} \phi .
\end{aligned}
$$

By taking the partial derivative in time of (2.5) and subtracting it with the partial derivative of (2.6), we get, using the Poisson equation,

$$
\partial_{x}\left(\left[n_{i}+\frac{n_{e}}{\varepsilon^{2}}+\delta^{2} \partial_{t}^{2}\right] E\right)=\partial_{x}^{2}\left(S_{i}-\frac{S_{e}}{\varepsilon^{2}}\right) .
$$

Providing some additional conditions on the initial conditions, one can show that we can equivalently use the Poisson equation or (2.7).

The scheme presented hereafter was introduced by Belaouar, Crouseilles, Degond and Sonnendrücker [1] for the resolution of the one species Vlasov Poisson system with a semi-Lagrangian method. The two species case was treated in [7] with a PIC method. Let us discretize equation (2.7) semi-implicitly in time,

$$
\partial_{x}\left(\left[n_{i}^{(m)}+\frac{n_{e}^{(m)}}{\varepsilon^{2}}\right] E^{(m+1)}+\frac{\delta^{2}}{(\Delta t)^{2}}\left(E^{(m+1)}-2 E^{(m)}+E^{(m-1)}\right)\right)=\partial_{x}^{2}\left(S_{i}^{(m)}-\frac{S_{e}^{(m)}}{\varepsilon^{2}}\right) .
$$

Now, in the last two terms of the left-hand side, we can use the Poisson equation at time $m \Delta t$ and $(m-1) \Delta t$ to get,

$$
\partial_{x}\left(\left[n_{i}^{(m)}+\frac{n_{e}^{(m)}}{\varepsilon^{2}}+\frac{\delta^{2}}{(\Delta t)^{2}}\right] E^{(m+1)}\right)=\partial_{x}^{2}\left(S_{i}^{(m)}-\frac{S_{e}^{(m)}}{\varepsilon^{2}}\right)+\frac{2\left(n_{i}^{(m)}-n_{e}^{(m)}\right)-\left(n_{i}^{(m-1)}-n_{e}^{(m-1)}\right)}{(\Delta t)^{2}} .
$$

In order to rewrite the right-hand side we can approach $\left.\partial_{t}\left(n_{i}-n_{e}\right)\right|_{t=m \Delta t}$ with

$$
\frac{\left(n_{i}^{(m)}-n_{e}^{(m)}\right)-\left(n_{i}^{(m-1)}-n_{e}^{(m-1)}\right)}{\Delta t} .
$$


Then, using equation (2.5), we get,

$$
\partial_{x}\left(\left[n_{i}^{(m)}+\frac{n_{e}^{(m)}}{\varepsilon^{2}}+\frac{\delta^{2}}{(\Delta t)^{2}}\right] E^{(m+1)}\right)=\partial_{x}^{2}\left(S_{i}^{(m)}-\frac{S_{e}^{(m)}}{\varepsilon^{2}}\right)+\frac{n_{i}^{(m)}-n_{e}^{(m)}}{(\Delta t)^{2}}-\frac{\partial_{x}\left(j_{i}^{(m)}-\frac{j_{e}^{(m)}}{\varepsilon}\right)}{\Delta t} .
$$

Now, for clarity, let us define,

$$
\begin{gathered}
a(x)=(\Delta t)^{2}\left(n_{i}^{(m)}+\frac{n_{e}^{(m)}}{\varepsilon^{2}}\right)+\delta^{2}, \\
b(x)=(\Delta t)^{2}\left(S_{i}^{(m)}-\frac{S_{e}^{(m)}}{\varepsilon^{2}}\right), \\
c(x)=n_{i}^{(m)}-n_{e}^{(m)}, \\
d(x)=-\Delta t\left(j_{i}^{(m)}-\frac{j_{e}^{(m)}}{\varepsilon}\right) .
\end{gathered}
$$

At this point we need to solve the following equation,

$$
\partial_{x}\left(a E^{(m+1)}\right)=\partial_{x}^{2} b+c+\partial_{x} d .
$$

We integrate numerically equation (2.8) on the uniform mesh $\left\{x_{0}, \ldots, x_{n-2}\right\}$ (with $x_{n-1}=x_{0}$ ) with the space step $\Delta x$. Between $x_{0}$ et $x_{k}$, it gives,

$$
a_{k} E_{k}-a_{0} E_{0}=\left.\partial_{x} b\right|_{x=x_{k}}-\left.\partial_{x} b\right|_{x=x_{0}}+\sum_{j=0}^{k-1} c_{j} \Delta x+d_{k}-d_{0}
$$

which is approached by,

$$
a_{k} E_{k}-a_{0} E_{0}=\frac{b_{k+1}-b_{k}}{\Delta x}-\frac{b_{1}-b_{0}}{\Delta x}+\sum_{j=0}^{k-1} c_{j} \Delta x+d_{k}-d_{0}
$$

and then,

$$
E_{k}=\frac{1}{a_{k}}\left(a_{0} E_{0}+\frac{b_{k+1}-b_{k}}{\Delta x}-\frac{b_{1}-b_{0}}{\Delta x}+\sum_{j=0}^{k-1} c_{j} \Delta_{x}+d_{k}-d_{0}\right) .
$$

We need an additional equation to close the system. We know that the electric field $E$ has zero mean value since it derives from a potential. Therefore, the following equation holds,

$$
\sum_{k=0}^{n-2} E_{k}=0
$$

which gives $E_{0}$. Indeed,

$$
-a_{0} E_{0} \sum_{k=0}^{n-2} \frac{1}{a_{k}}=\sum_{k=0}^{n-2}\left(E_{k}-\frac{a_{0} E_{0}}{a_{k}}\right)=\sum_{k=0}^{n-2} \frac{1}{a_{k}}\left(\frac{b_{k+1}-b_{k}}{\Delta x}-\frac{b_{1}-b_{0}}{\Delta x}+\sum_{j=0}^{k-1} c_{j} \Delta_{x}+d_{k}-d_{0}\right)
$$


and so,

with,

$$
E_{0}=\left(a_{0} \Sigma_{1}\right)^{-1} \times\left(-\frac{\Sigma_{2}}{\Delta x}+\frac{b_{1}-b_{0}}{\Delta x} \Sigma_{1}-\Sigma_{3} \Delta x-\Sigma_{4}+d_{0} \Sigma_{1}\right)
$$

$$
\Sigma_{1}=\sum_{k=0}^{n-2} \frac{1}{a_{k}}, \quad \Sigma_{2}=\sum_{k=0}^{n-2} \frac{b_{k+1}-b_{k}}{a_{k}}, \quad \Sigma_{3}=\sum_{k=0}^{n-2} \sum_{j=0}^{k-1} \frac{c_{j}}{a_{k}}, \quad \Sigma_{4}=\sum_{k=0}^{n-2} \frac{d_{k}}{a_{k}} .
$$

The asymptotic preserving Poisson solver is adapted to large scale regimes and we refer to [1] for relevant test cases. In the next section, we shall focus on micro kinetic phenomena in the massless electron asymptotic. For this purpose, we will use a classical Poisson solver, setting $\delta=1$.

\section{NumericAl ReSults}

We want to investigate the massless electron approximation for our modelling. In our initial modelling we neglected the collisions between particles. However in the small mass approximation, namely $\varepsilon \rightarrow 0$, we can infer from equation (1.3) that the time scales of the electrons diminish and their dynamic accelerates. Therefore the collisionless hypothesis is questionable in this asymptotic. Let us suppose, crudely, that because of collisions the electron density is at thermodynamical equilibrium, namely,

$$
f_{e}(t, x, v)=n_{e}(t, x) M(v),
$$

where $M$ is the rescaled Maxwellian,

$$
M(v)=\frac{1}{\sqrt{2 \pi}} e^{-\frac{v^{2}}{2}}
$$

Injecting this expression in the electron equation of (1.4) yields,

$$
\varepsilon \partial_{t} n_{e}+v\left(\partial_{x} n_{e}+E n_{e}\right)=0 .
$$

Letting $\varepsilon$ goes to zero gives formally,

$$
\partial_{x} n_{e}+E n_{e}=0 .
$$

since the equality before the last hold for any $v$. Now, we can use the fact that $E=-\partial_{x} \phi$ to get,

$$
n_{e}(t, x)=C(t) e^{\phi(t, x)},
$$

with

$$
C(t)=\frac{\int n_{i} d x}{\int e^{\phi} d x}
$$

because of the global neutrality. The goal of the following numerical test cases is to determine whether the electron density gets close to the latter rescaled Maxwell-Boltzmann density as $\varepsilon$ gets small, when following the two-species non-collisional equation (1.4). We will suppose that the initial electron density is at global equilibria, namely

$$
f_{e}(0, x, v)=M(v),
$$

whereas the ions will be such that they impose their dynamic on the coupled system. Actually their initial density will be of the following form,

$$
f_{i}(0, x, v)=\left(1+A \cos \left(\frac{2 \pi}{L} x\right)\right) \nu(v),
$$

where $A \ll 1$ is the amplitude of the perturbation. We will try two velocity profiles $\nu$, one being stable and the other being unstable in the sense of Penrose (see Definition 3.1). 
Definition 3.1 ( $[13])$. We say that an homogeneous even profile $\nu(v)$, such that $\int \nu d v=1$, satisfies the Penrose instability criterion if $\mu$ has a local minimum point in 0 and,

$$
\int_{\mathbb{R}} \frac{\nu(v)-\nu(0)}{v^{2}} d v>\frac{4 \pi^{2}}{L^{2}}
$$

Under some additional smoothness properties for the profile $\nu$, Guo and Strauss proved the equivalence between the Penrose condition and the nonlinear instability of $\nu$ for the one species Vlasov Poisson system [11]. The kinetic instabilities created by these profiles are often referred to as two-stream instabilities, because they appear for $\nu$ with two or more maxima.

In order to measure the distance between $n_{e}$ associated with a solution of (1.4) and the Maxwell-Boltzmann density, we introduce the following quantity,

$$
d(t)=\frac{1}{L}\left\|n_{e}(t, \cdot)-C(t) e^{\phi(t, \cdot)}\right\|_{L_{x}^{2}}^{2} .
$$

\subsection{Test case 1: stability}

In this first test case, we consider a grid of 512 points in the $x \in[0,21]$ direction and of 512 points in the $v \in[-7,7]$ direction. The time step is $\Delta t=0.01$ and we stop the simulation at $t_{\max }=15$. The amplitude of the perturbation is $A=0.001$ and the ion velocity profile is the following Maxwellian distribution,

$$
\nu(v)=M(v) .
$$

We perform the simulation of system (1.4) for the following mass ratio,

$$
\varepsilon \in\{1,0.5,0.1,0.05,0.01,0.005\}
$$

The error on the conservation of mass, total energy and entropy can be found in Table 1.

\begin{tabular}{|r|c|c|c|c|c|c|}
\hline$\varepsilon=$ & 1 & 0.5 & 0.1 & 0.05 & 0.01 & 0.005 \\
\hline Mass (ions): $\iint f_{i} d v d x$ & $10^{-12}$ & $10^{-12}$ & $10^{-12}$ & $10^{-12}$ & $10^{-12}$ & $10^{-12}$ \\
\hline Mass (electrons): $\iint f_{e} d v d x$ & $10^{-12}$ & $10^{-12}$ & $10^{-12}$ & $10^{-12}$ & $10^{-10}$ & $10^{-9}$ \\
\hline Total energy: $\mathcal{E}(t)$ & $10^{-8}$ & $10^{-9}$ & $10^{-8}$ & $10^{-9}$ & $10^{-8}$ & $10^{-8}$ \\
\hline Entropy: $\mathcal{S}_{i}(t)+\mathcal{S}_{e}(t)$ & $10^{-12}$ & $10^{-12}$ & $10^{-12}$ & $10^{-12}$ & $10^{-11}$ & $10^{-10}$ \\
\hline
\end{tabular}

TABle 1. Test case 1: stability. Relative error on mass, total energy and entropy at time $t_{\max }$ for different values of $\varepsilon$.

As we can observe on Figure 2, in this stable case, the macroscopic electron density is exponentially damped in time towards the Maxwell-Boltzmann density with a rate that increases as the mass ratio $\varepsilon$ diminishes. The accuracy of this simulation is confirmed by the weak relative error on the control variates of Table 1 .

\subsection{Test case 2: instability}

In this second test case, we consider a grid of 512 points in the $x \in[0,21]$ direction and of 2048 points in the $v \in[-5,5]$ direction. The time step is $\Delta t=0.02$ and we stop the simulation at $t_{\max }=100$. The amplitude of the perturbation is $A=0.01$ and the ion velocity profile is the following two-stream density,

$$
\nu(v)=8 v^{2} M(2 v),
$$


which satisfies the Penrose instability criterion of Definition 3.1. We perform the simulation of system (1.4) for the following mass ratio,

$$
\varepsilon \in\{1,0.5,0.1,0.05,0.01,0.005\}
$$

The error on the conservation of mass, total energy and entropy can be found in Table 2.

\begin{tabular}{|r|c|c|c|c|c|c|}
\hline$\varepsilon=$ & 1 & 0.5 & 0.1 & 0.05 & 0.01 & 0.005 \\
\hline Mass (ions) & $4.1 \times 10^{-3}$ & $4.2 \times 10^{-3}$ & $3.2 \times 10^{-3}$ & $2.9 \times 10^{-3}$ & $2.2 \times 10^{-3}$ & $3.2 \times 10^{-3}$ \\
\hline Mass (electrons) & $5.2 \times 10^{-4}$ & $5.3 \times 10^{-4}$ & $5.3 \times 10^{-5}$ & $7.1 \times 10^{-5}$ & $1.0 \times 10^{-5}$ & $1.2 \times 10^{-4}$ \\
\hline Total energy & $7.0 \times 10^{-4}$ & $6.9 \times 10^{-4}$ & $6.0 \times 10^{-4}$ & $5.4 \times 10^{-4}$ & $4.2 \times 10^{-4}$ & $3.9 \times 10^{-4}$ \\
\hline Entropy & $3.2 \times 10^{-3}$ & $4.7 \times 10^{-3}$ & $3.3 \times 10^{-3}$ & $3.0 \times 10^{-3}$ & $2.7 \times 10^{-3}$ & $3.2 \times 10^{-3}$ \\
\hline
\end{tabular}

TABLe 2. Test case 2: instability. Relative error on mass, total energy and entropy at time $t_{\max }$ for different values of $\varepsilon$.

We observe on Figure 3 that in this case, there does not seem to be any convergence to the Maxwell-Boltzmann density neither in time nor in $\varepsilon$. Moreover, as witnesses Figures 4 and 5 , the two-stream instability arises at similar time and with comparable electric energy amplitude, whatever the value of $\varepsilon$. Therefore, the smallness of the mass ratio does not seem to prevent from the creation of micro kinetic instabilities such as two-stream instabilities. Compared to the first test case, the relative errors on the control variates in Table 2 are larger but uniform in $\varepsilon$. We can infer that this is due to the turbulent dynamic and the fact that the simulation is longer.

\section{CONCLUSION}

In this paper, we proposed a rescaled kinetic modelling of a two species plasma by taking the ion dynamic as reference. This allowed us to introduce two important asymptotics in this context, namely the quasineutral limit and the massless electron approximation. In a one dimensional non-magnetized framework, we adapted a one species numerical scheme to our problem and a Poisson solver that can, according to its designers, handle the first asymptotic. Finally we illustrated the zero mass ratio limit with numerical simulations of a two species plasma in the non quasineutral regime. With accurate numerical experiments, we demonstrated the incompatibility between the existence of microkinetic instabilities at the two-species level with the MaxwellBoltzmann approximation for electrons.

ACKNOWLEDGEMENTS. The authors would like to acknowledge the organizers of the CEMRACS 2014 and the staff of the CIRM for making this work possible. Also, both authors would like to thank their supervisors on this project, Francis Filbet for the advising and many insightful comments and Morgane Bergot for the help during the realisation. This work has been carried out within the framework of the EUROfusion Consortium and has received funding from the Euratom research and training programme 2014-2018 under grant agreement No 633053. The views and opinions expressed herein do not necessarily reflect those of the European Commission. Furthermore, Maxime Herda is partially supported by the European Research Council ERC Starting Grant 2009, project 239983-NuSiKiMo.

\section{REFERENCES}

[1] R. Belaouar, N. Crouseilles, P. Degond, and E. Sonnendrücker. An asymptotically stable semi-Lagrangian scheme in the quasi-neutral limit. J. Sci. Comput., 41(3):341-365, 2009.

[2] Paul Murray Bellan. Fundamentals of plasma physics. Cambridge University Press, 2006.

[3] Charles K Birdsall and A Bruce Langdon. Plasma physics via computer simulation. CRC Press, 2004.

[4] F. Bouchut and J. Dolbeault. On long time asymptotics of the Vlasov-Fokker-Planck equation and of the Vlasov-PoissonFokker-Planck system with Coulombic and Newtonian potentials. Differential Integral Equations, 8(3):487-514, 1995.

[5] François Bouchut. Global weak solution of the Vlasov-Poisson system for small electrons mass. Comm. Partial Differential Equations, 16(8-9):1337-1365, 1991. 
[6] François Bouchut, François Golse, and Mario Pulvirenti. Kinetic equations and asymptotic theory, volume 4 of Series in Applied Mathematics (Paris). Gauthier-Villars, Éditions Scientifiques et Médicales Elsevier, Paris, 2000. Edited and with a foreword by Benoît Perthame and Laurent Desvillettes.

[7] Pierre Degond, Fabrice Deluzet, Laurent Navoret, An-Bang Sun, and Marie-Hélène Vignal. Asymptotic-preserving particle-incell method for the Vlasov-Poisson system near quasineutrality. J. Comput. Phys., 229(16):5630-5652, 2010.

[8] F. Filbet and E. Sonnendrücker. Comparison of Eulerian Vlasov solvers. Comput. Phys. Comm., 150(3):247-266, 2003.

[9] Emmanuel Frénod and Eric Sonnendrücker. Homogenization of the Vlasov equation and of the Vlasov-Poisson system with a strong external magnetic field. Asymptot. Anal., 18(3-4):193-213, 1998.

[10] François Golse and Laure Saint-Raymond. The Vlasov-Poisson system with strong magnetic field. J. Math. Pures Appl. (9), 78(8):791-817, 1999.

[11] Yan Guo and Walter A. Strauss. Nonlinear instability of double-humped equilibria. Ann. Inst. H. Poincaré Anal. Non Linéaire, 12(3):339-352, 1995.

[12] Daniel Han-Kwan. Quasineutral limit of the Vlasov-Poisson system with massless electrons. Comm. Partial Differential Equations, 36(8):1385-1425, 2011.

[13] Daniel Han-Kwan and Maxime Hauray. Stability Issues in the Quasineutral Limit of the One-Dimensional Vlasov-Poisson Equation. Comm. Math. Phys., 334(2):1101-1152, 2015.

[14] Kenro Miyamoto. Plasma physics and controlled nuclear fusion, volume 38. Springer, 2006.

[15] H. Neunzert and J. Wick. The convergence of simulation methods in plasma physics. In Mathematical methods of plasmaphysics (Oberwolfach, 1979), volume 20 of Methoden Verfahren Math. Phys., pages 271-286. Lang, Frankfurt, 1980.

[16] L. Saint-Raymond. The gyrokinetic approximation for the Vlasov-Poisson system. Math. Models Methods Appl. Sci., 10(9):1305-1332, 2000.

[17] Chang Yang and Francis Filbet. Conservative and non-conservative methods based on Hermite weighted essentially nonoscillatory reconstruction for Vlasov equations. J. Comput. Phys., 279:18-36, 2014.

This is an Open Access article distributed under the terms of the Creative Commons Attribution License (http://creativecommons.org/licenses/by/4.0), which permits unrestricted use, distribution, and reproduction in any medium, provided the original work is properly cited. 
ESAIM: PROCEEDINGS AND SURVEYS

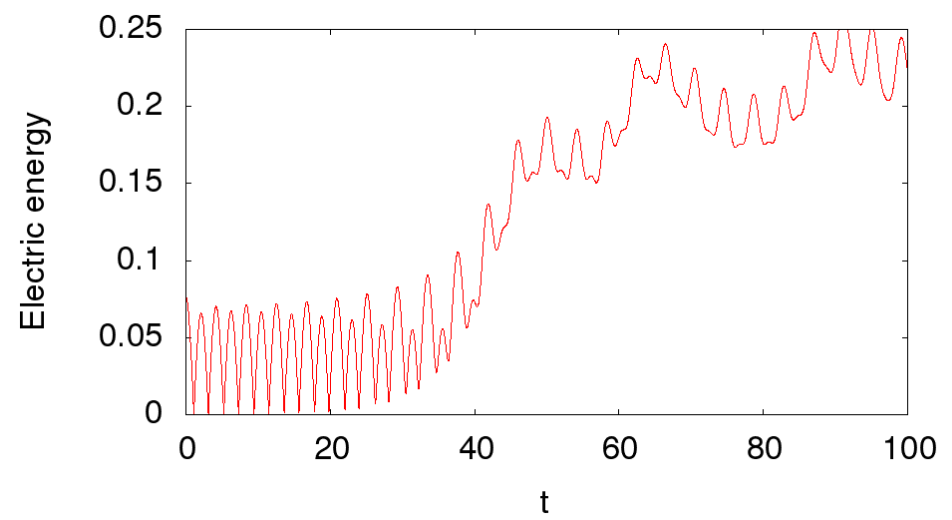

(a) $\varepsilon=1$

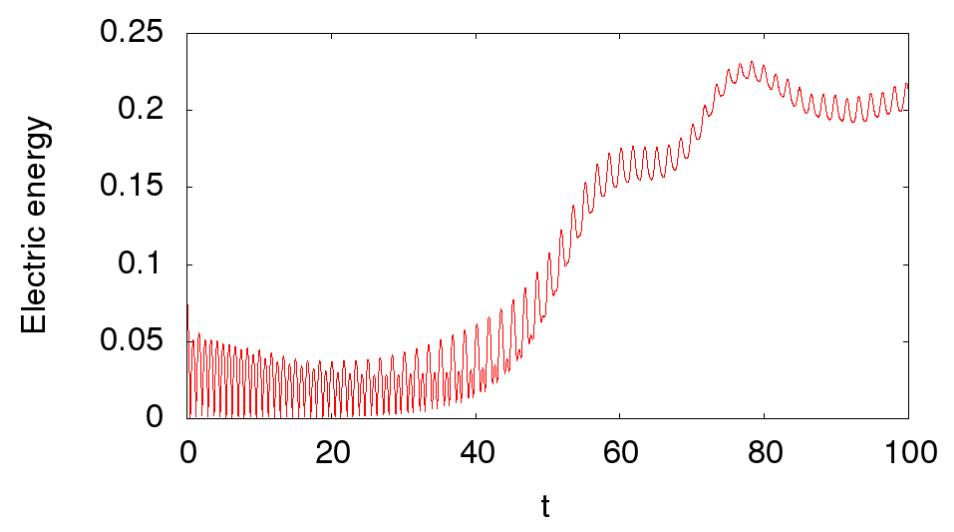

(c) $\varepsilon=0.1$

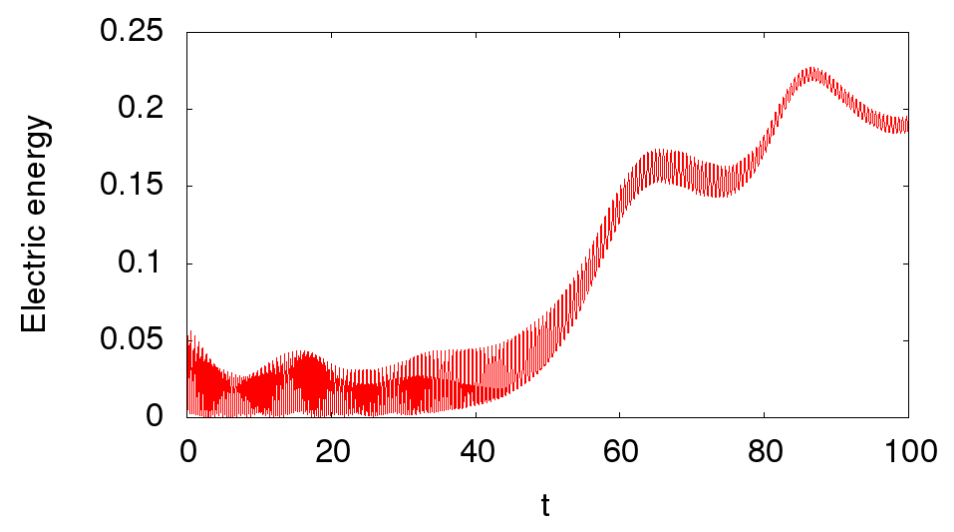

(e) $\varepsilon=0.01$

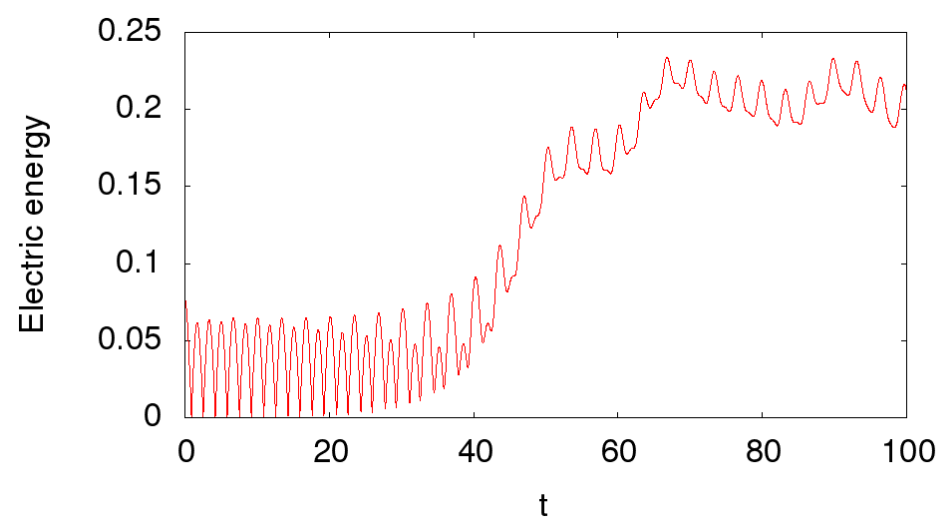

(b) $\varepsilon=0.5$

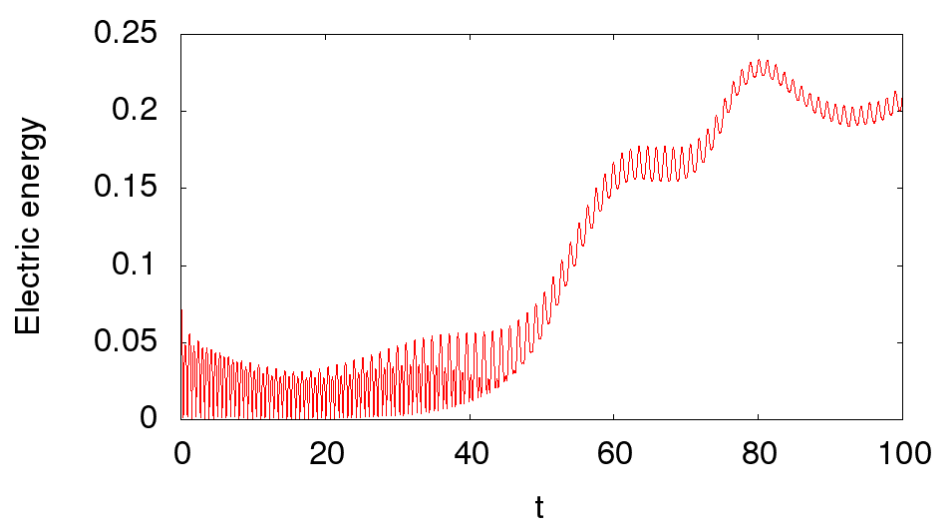

(d) $\varepsilon=0.05$

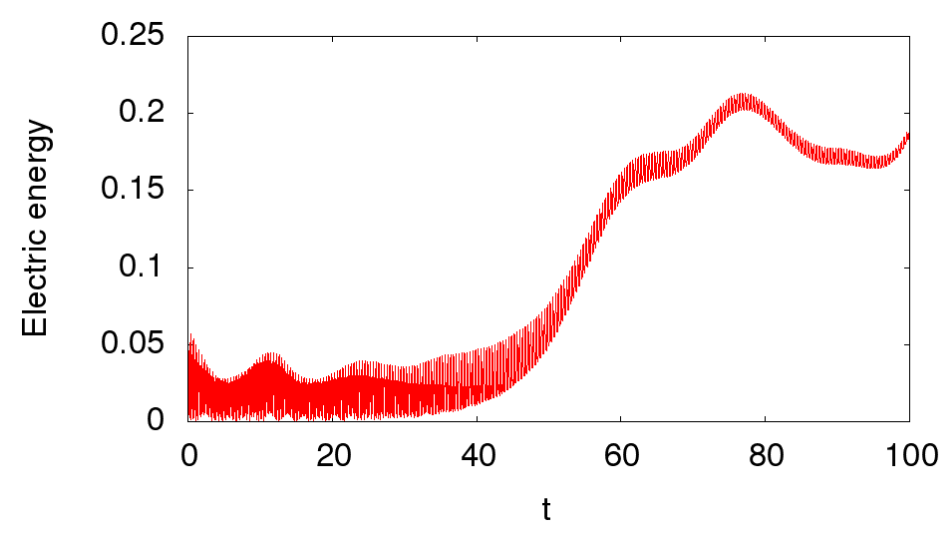

(f) $\varepsilon=0.005$

Figure 4. Test case 2: instability. Time evolution of the electric energy $\frac{1}{2} \int\left|\partial_{x} \phi\right|^{2} d x$ for different values of $\varepsilon$. 


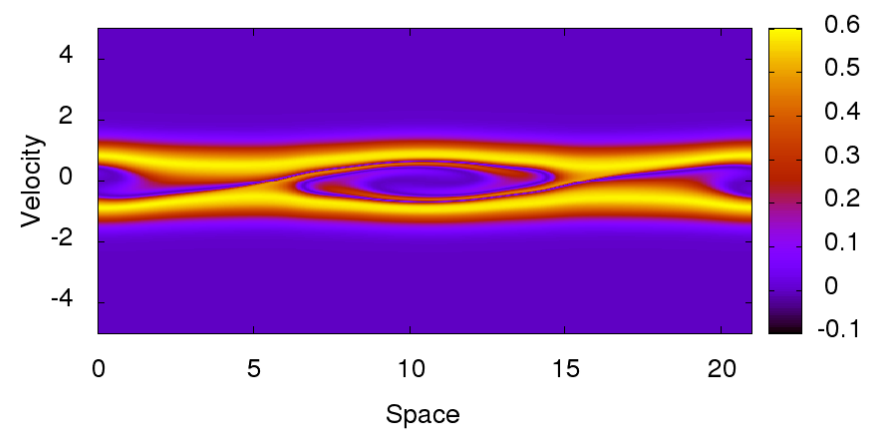

(a) $\varepsilon=1$

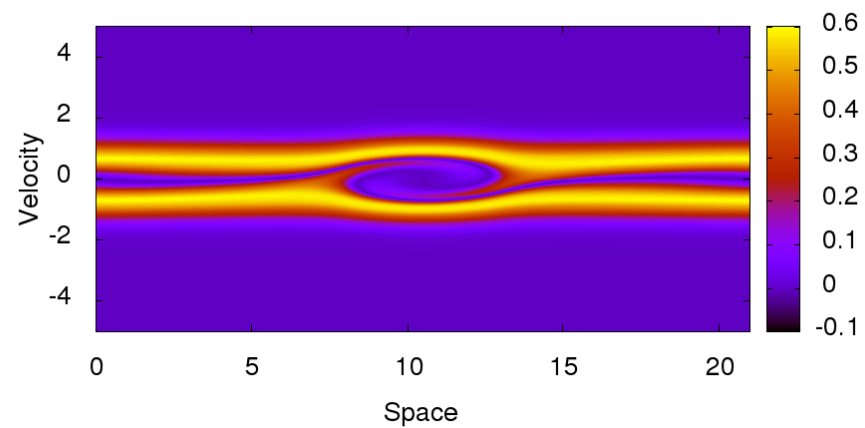

(c) $\varepsilon=0.1$

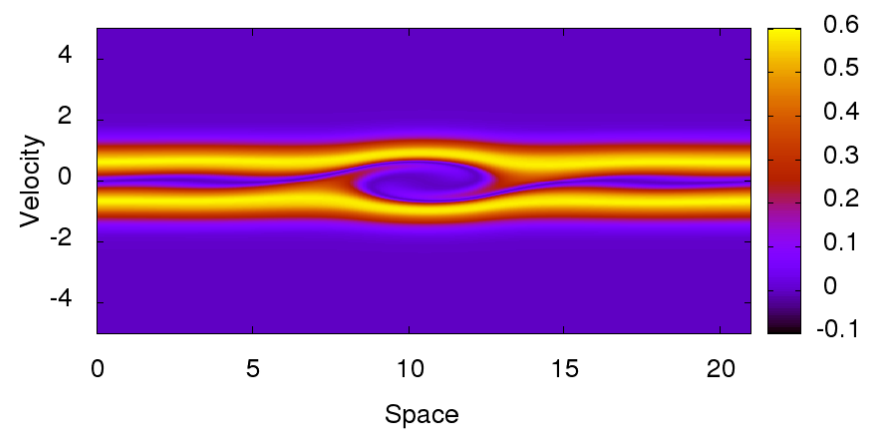

(e) $\varepsilon=0.005$

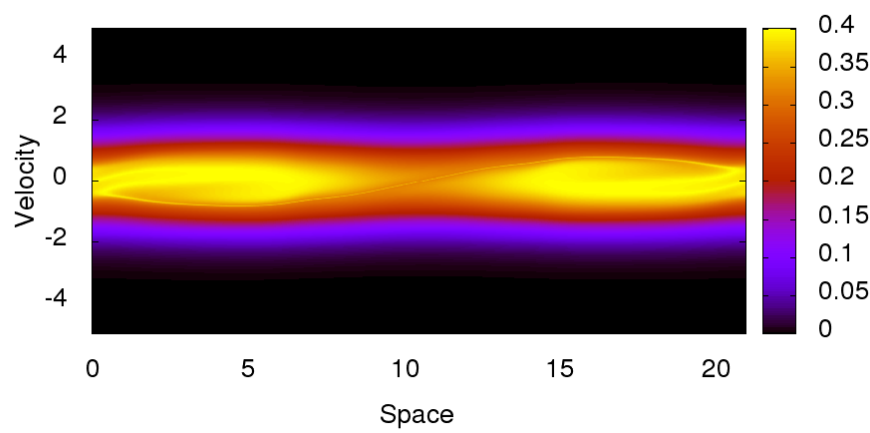

(b) $\varepsilon=1$

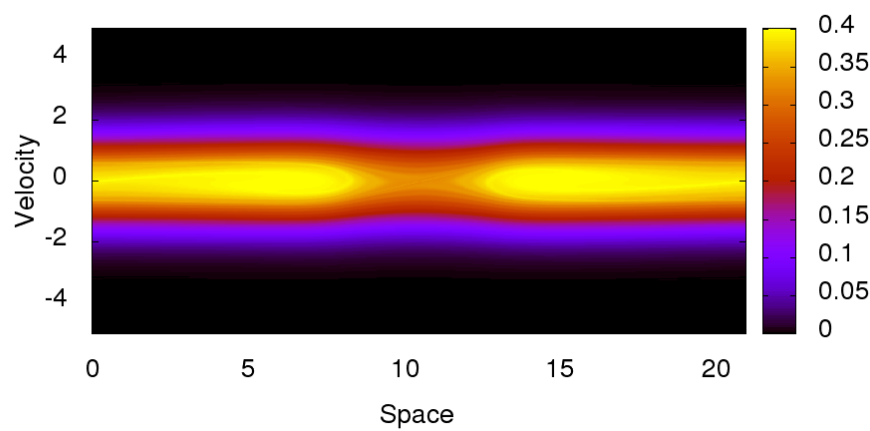

(d) $\varepsilon=0.1$

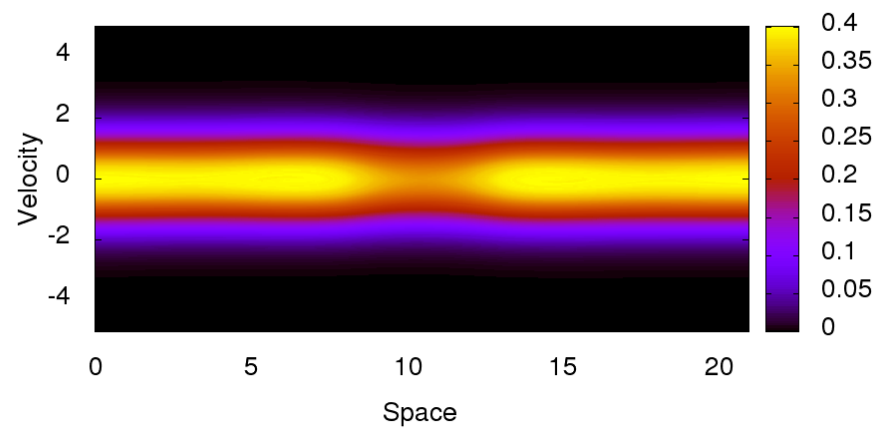

(f) $\varepsilon=0.005$

Figure 5. Test case 2: instability. Densities during the creation of the instability $(t=60$; Left: Ions; Right: Electrons) for different values of $\varepsilon$. 